\title{
Resultados da transferência do grande dorsal alongado com enxerto tendíneo homólogo por via delto-peitoral para lesões póstero-superiores irreparáveis do manguito rotador
}

\section{Results of Latissimus Dorsi Transfer using a Tendinous Allograft through a Single Deltopectoral Approach for Irreparable Posterosuperior Rotator Cuff Tears}

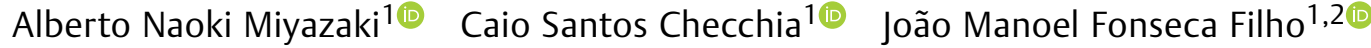

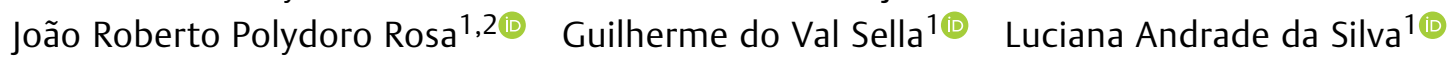 \\ ${ }^{1}$ Grupo de Cirurgia em Ombro e Cotovelo, Departamento de \\ Ortopedia e Traumatologia, Faculdade de Ciências Médicas, Santa \\ Casa de São Paulo, São Paulo, SP, Brasil \\ ${ }^{2}$ Departamento de Cirurgia Ortopédica, Hospital Alemão Oswaldo \\ Cruz, São Paulo, SP, Brasil \\ Endereço para correspondência Caio Santos Checchia, Grupo de \\ Ombro e Cotovelo, Departamento de Ortopedia e Traumatologia, \\ Faculdade de Ciências Médicas, Santa Casa de São Paulo, Rua Dr. \\ Cesário Mota Junior, 112, Consolação, São Paulo, SP, 01221-020, Brasil \\ (e-mail: caio.checchia@gmail.com).
}

Rev Bras Ortop 2022;57(4):590-598.

Resumo

\section{Palavras-chave}

- lesões do manguito rotador

- transferência de tendão

- ombro

- procedimentos ortopédicos
Objetivo O objetivo primário do presente trabalho é avaliar os resultados funcionais de uma modificação na transferência do grande dorsal no ombro para o tratamento de lesões póstero-superiores irreparáveis do manguito rotador. O objetivo secundário é avaliar as variáveis que podem influenciar os resultados.

Técnica cirúrgica Por meio de uma única abordagem deltopeitoral, o tendão do músculo grande dorsal é desinserido reforçado e alongado com um enxerto tendíneo homólogo, transferido para o úmero e fixado em posição superolateral ao tubérculo maior e anterior ao músculo subescapular.

Métodos Avaliação funcional retrospectiva de 16 casos. O período médio de acompanhamento foi de 21 meses (12-47 meses). Os resultados pós-operatórios (no último acompanhamento) foram comparados aos pré-operatórios, bem como a outras variáveis pré, intra e pós-operatórias.

Resultados Todos os pacientes ficaram satisfeitos (exceto um). A pontuação média da University of California, Los Angeles (UCLA) aumentou de 11,6 (8-16) para 27,3 (17-30)
Estudo desenvolvido no Grupo de Cirurgia em Ombro e Cotovelo do Departamento de Ortopedia e Traumatologia da Faculdade de Ciências Médicas da Santa Casa de São Paulo, São Paulo, Brasil. recebido

07 de Agosto de 2020

aceito

02 de Outubro de 2020

Publicado on-line

Março 31, 2021
DOI https://doi.org/

10.1055/s-0041-1724073.

ISSN 0102-3616.
(C) 2021. Sociedade Brasileira de Ortopedia e Traumatologia. All rights reserved.

This is an open access article published by Thieme under the terms of the Creative Commons Attribution-NonDerivative-NonCommercial-License, permitting copying and reproduction so long as the original work is given appropriate credit. Contents may not be used for commercial purposes, or adapted, remixed, transformed or built upon. (https://creativecommons.org/ licenses/by-nc-nd/4.0/)

Thieme Revinter Publicações Ltda., Rua do Matoso 170, Rio de Janeiro, RJ, CEP 20270-135, Brazil 


Abstract

Keywords
- rotator cuff injuries
- tendon transfer
- shoulder
- orthopedic
procedures

$(p<0,001)$. A dor, a função e a força do ombro apresentaram melhora estatisticamente significativa $(p<0,001)$. A força, porém, não voltou ao valor normal. A amplitude de movimento ativa média apresentou as seguintes melhoras: elevação frontal, de $106^{\circ}$ $\left(60-140^{\circ}\right)$ para $145^{\circ}\left(130-160^{\circ}\right)(p<0,001)$; rotação externa, de $30^{\circ}\left(0-60^{\circ}\right)$ para $54^{\circ}$ $\left(40-70^{\circ}\right)(p<0,001)$; e rotação interna, de L1 (glúteo a T7) para T10 (T12-T3) $(p<0,05)$. Nenhuma complicação foi observada. A pseudoparesia pré-operatória foi revertida em todos os seis casos em que foi observada. Nenhuma das variáveis analisadas influenciou os desfechos, nem mesmo a pseudoparesia.

Conclusões A curto prazo essa técnica é segura e eficaz na recuperação da pseudoparesia e na melhora da dor, da função e da força do ombro.

Objective The primary aim of the present study is to evaluate the functional results of a modification to the latissimus dorsi (LD) transfer around the shoulder for irreparable posterosuperior rotator cuff tears. The secondary aim is to evaluate variables that might influence the outcomes.

Surgical Technique Through a single deltopectoral approach, the LD tendon is detached, reinforced, and elongated with a tendinous allograft, transferred around the humerus, and fixed superolaterally to the greater tuberosity and anteriorly to the subscapularis.

Methods Retrospective functional evaluation of 16 cases. The average follow-up was 21 months (12-47). The postoperative results (at last follow-up) were compared with the preoperative ones, as well as to other pre, intra, and postoperative variables.

Results All (but one) patients were satisfied. Average University of California, Los Angeles (UCLA) score increased from $11.6(8-16)$ to $27.3(17-30)(p<0.001)$. Improvements of shoulder pain, function, and strength achieved statistical significance $(p<0.001)$. Nonetheless, normal strength was never restored. Average active range of motion improved as follows: forward elevation, from $106^{\circ}\left(60-140^{\circ}\right)$ to $145^{\circ}(130-$ $\left.160^{\circ}\right)(p<0.001)$; external rotation from $30^{\circ}\left(0^{\circ}\right.$ to $\left.60^{\circ}\right)$ to $54^{\circ}\left(40-70^{\circ}\right)(p<0,001)$; and internal rotation from L1 (gluteus to T7) to T10 (T12-T3) $(p<0.05)$. No complication has occurred. Preoperative pseudoparesis was reverted in all the six cases in which it was present. None of the variables analyzed influenced the outcomes, including pseudoparesis.

\section{Introdução}

Lesões crônicas irreparáveis do tendão do manguito rotador provocam atrofia, infiltração gordurosa e comprometimento funcional dos respectivos ventres musculares. ${ }^{1}$ Ao afetarem a porção póstero-superior do manguito rotador (tendões supra-espinal e infra-espinal), causam principalmente dor, redução da força para rotação externa e elevação do ombro e dificuldade de posicionamento da mão no espaço. ${ }^{1,2}$

Embora essas lesões não possam ser reparadas, diversas técnicas cirúrgicas foram descritas para melhorar a função do ombro. A artroplastia total reversa do ombro teve resultados funcionais bons e previsíveis no acompanhamento em curto e médio prazo. ${ }^{3}$ Ainda assim, dois pontos importantes sugerem que esse procedimento deve ser reservado para populações mais idosas (pacientes com 65 anos ou mais): (1) há evidências de que, 10 anos após a cirurgia, esses bons resultados funcionais se deterioram e as taxas de complicações aumentam de forma significativa; ${ }^{4}(2)$ a artroplastia total reversa do ombro tem sido bastante utilizada por um período relativamente curto (desde 2004 nos Estados Uni$\operatorname{dos}^{5}$ ) e, assim, há poucas evidências de seus resultados em longo prazo.

Por isso, outras técnicas são recomendadas para pacientes mais jovens e ativos. ${ }^{6}$ As três técnicas mais usadas são: (1) transferência do tendão do grande dorsal (TGD) para o tubérculo maior; ${ }^{1,2,7-13}$ (2) reconstrução capsular superior, ${ }^{14,15}$ e (3) transferência da porção inferior do trapézio para o tubérculo maior. ${ }^{16,17}$

Apesar de algumas publicações recentes sobre os desfe$\operatorname{chos}^{14,15}$ da reconstrução capsular superior, seus resultados em médio e longo prazo ainda são desconhecidos. Da mesma forma, os trabalhos acerca das transferências da porção inferior do trapézio ainda são poucos e recentes. ${ }^{16,17}$

A TGD foi descrita pela primeira vez por Gerber et al. ${ }^{1}$ em 1992 e há diversas publicações sobre seus resultados, inclusive em longo prazo. ${ }^{2,11,18}$ Dentre elas, vários autores demonstraram a possibilidade de obter bons resultados. ${ }^{2,9-11,19,20}$ No 
entanto, houve até 36\% de insucessos clínicos (bem como 38\% de rupturas da transferência). ${ }^{21}$ Além disso, os achados sobre os fatores preditivos de maus resultados ainda são escassos e controversos. $^{2,4,22,23}$

Há, porém, evidências de que a maioria dos insucessos se deve a um dos seguintes motivos: (1) ruptura da transferência do tubérculo maior ${ }^{12,13}$ e (2) ruptura da origem do deltoide. ${ }^{24}$ Dessa maneira, alguns autores recentemente propuseram mudanças à técnica original de Gerber. A artroscopia $^{8,12,21}$ e a abordagem única, ${ }^{9}$ por exemplo, têm sido recomendadas para prevenir a violação do deltoide. A desinserção do grande dorsal junto com alguns fragmentos ósseos da cortical umeral tem sido recomendada para reforçar a cicatrização da transferência no tubérculo maior. ${ }^{13}$

Ainda assim, a literatura atual carece de uma técnica que considere a prevenção simultânea dessas duas complicações. Com isso em mente, propusemos e publicamos recentemente $^{25}$ as seguintes modificações à técnica cirúrgica: o TGD é alongado e reforçado com enxerto tendíneo homólogo permitindo que sua transferência seja realizada por abordagem deltopeitoral única.

O objetivo primário do presente trabalho é avaliar, por meio de uma análise retrospectiva de casos, os resultados funcionais em curto prazo dos ombros submetidos a essa técnica. O objetivo secundário é analisar possíveis variáveis pré e intraoperatórias que podem influenciar os resultados.

\section{Materiais e métodos}

A técnica cirúrgica utilizada e o protocolo de reabilitação seguido foram os mesmos publicados anteriormente. ${ }^{25} \mathrm{~A}$ técnica é resumida nas - Figs. 1-2 a 3.

Entre novembro de 2013 e agosto de 2018, a técnica citada $^{25}$ foi realizada em 20 pacientes com rupturas póstero-superiores irreparáveis do manguito rotador. Os pron- tuários médicos desses pacientes foram avaliados retrospectivamente quanto aos dados relacionados a achados clínicos e epidemiológicos pré e pós-operatórios.

A cirurgia foi proposta aos pacientes após o insucesso do tratamento conservador com fisioterapia e somente após explicar que não havia expectativa de recuperação funcional completa.

O diagnóstico foi estabelecido por ressonância magnética e confirmado à cirurgia. A infiltração gordurosa do subescapular, supraespinhoso e infraespinhoso, foi classificada de acordo com o método de Goutallier modificado por Fuchs et al. ${ }^{26}$ Da mesma maneira, as radiografias pré-operatórias foram usadas para classificação de Hamada. ${ }^{27}$

A irreparabilidade dos tendões foi determinada pela impossibilidade intraoperatória de sua redução ao tubérculo maior com o ombro a $45^{\circ}$ de abdução após a liberação tendínea adequada. ${ }^{6}$ Nenhum paciente deste estudo pôde ser submetido ao reparo parcial devido à retração do tendão.

A amplitude de movimentos (ADM) ativa e passiva antes e depois da cirurgia foi registrada de acordo com o método proposto pela American Academy of Orthopaedic Surgeons. ${ }^{28}$ As funções pré e pós-operatórias foram avaliadas de acordo com a pontuação da University of California, Los Angeles (UCLA) modificada por Ellman et al. ${ }^{29}$

Os critérios de inclusão foram pacientes adultos com lesões irreparáveis do manguito rotador tratados com esta técnica e acompanhamento pós-operatório mínimo de um ano. Os critérios de exclusão foram: não consentimento à participação no estudo, não adesão ao protocolo de reabilitação e cirurgia prévia no ombro.

Dos 20 pacientes operados, 16 atenderam aos critérios de inclusão (três foram excluídos devido ao curto período de acompanhamento e um paciente foi excluído por abandono do protocolo de reabilitação [por ter sofrido um acidente vascular cerebral três meses após a cirurgia]). Seis (37,5\%)

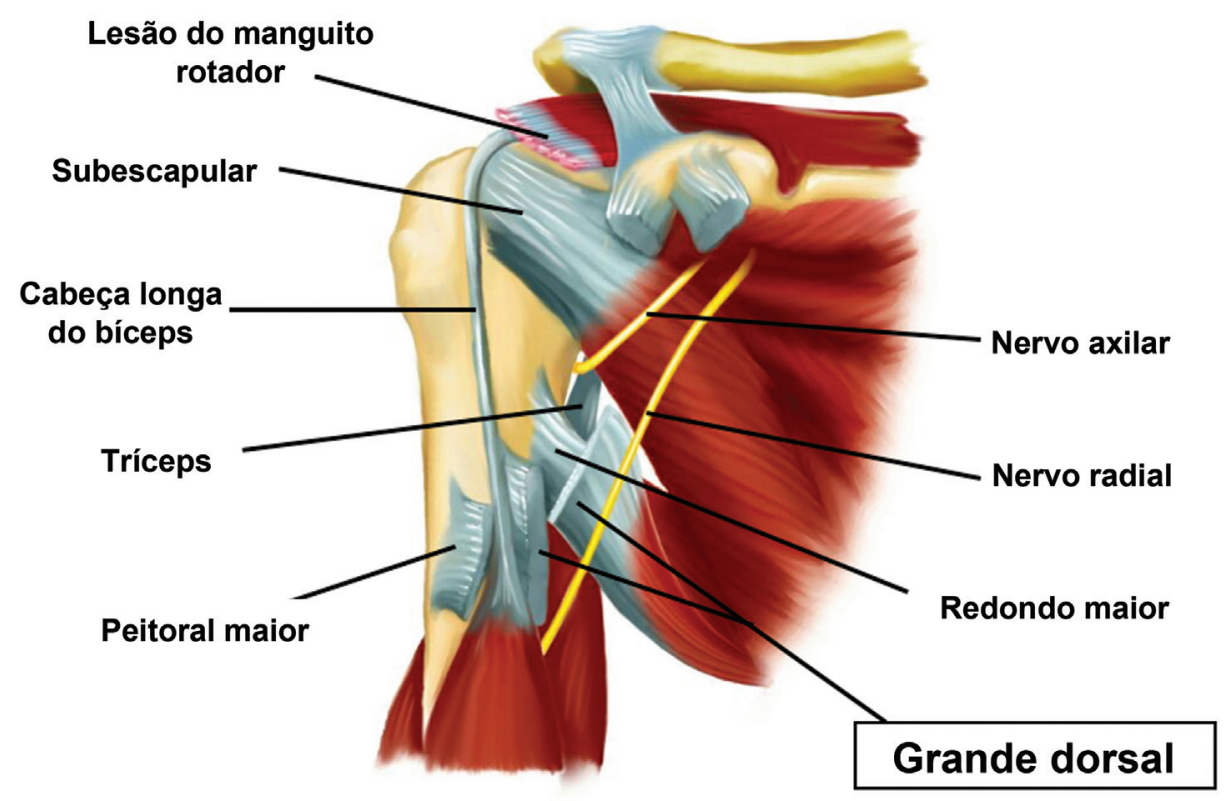

Fig. 1 Ilustração de um ombro direito (vista anterior) representando as estruturas anatômicas (o peitoral maior foi tenotomizado e afastado; o tendão do grande dorsal já foi desinserido do úmero). 


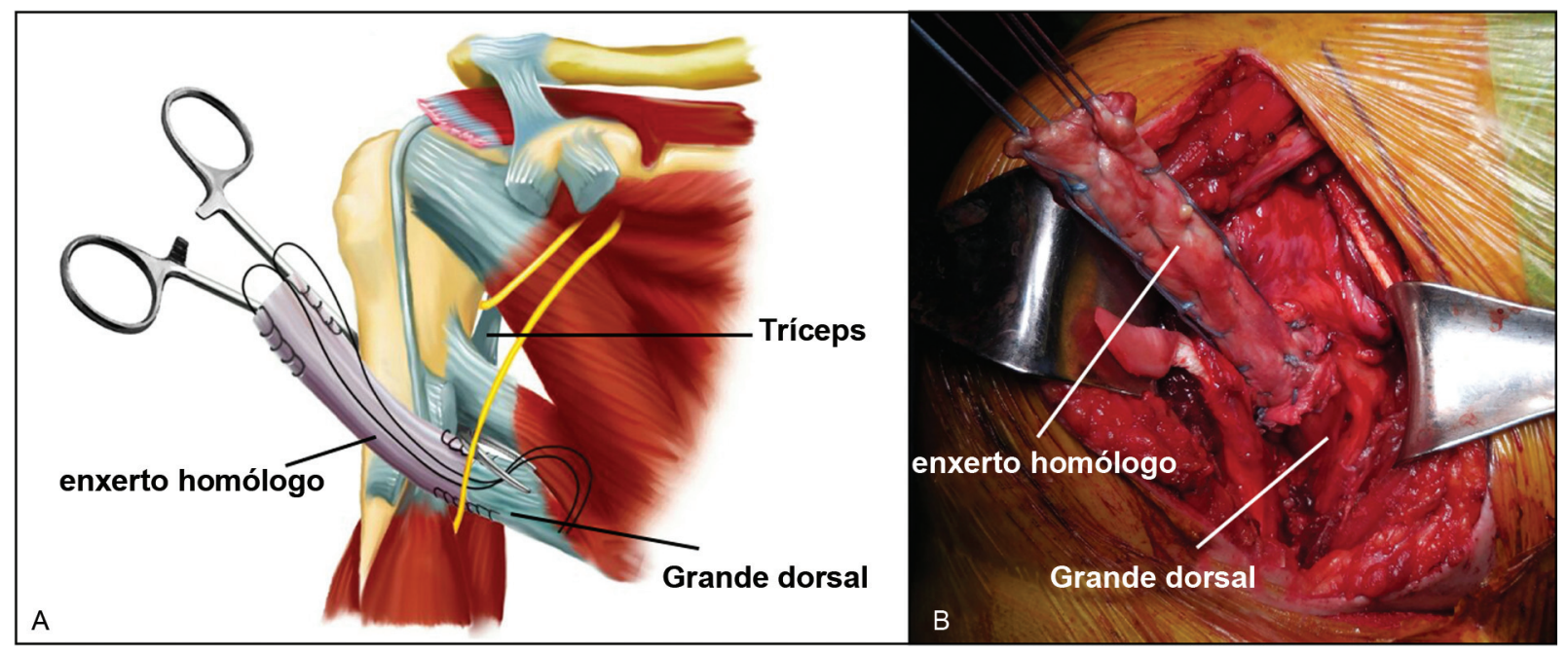

Fig. 2 (A) Ilustração de ombro direito (vista anterior), mostrando a etapa cirúrgica em que o enxerto tendíneo homólogo já foi suturado ao tendão do grande dorsal nativo e uma pinça cirúrgica foi passada posteriormente à diáfise umeral. (B) Fotografia intraoperatória do ombro direito durante esta etapa cirúrgica.

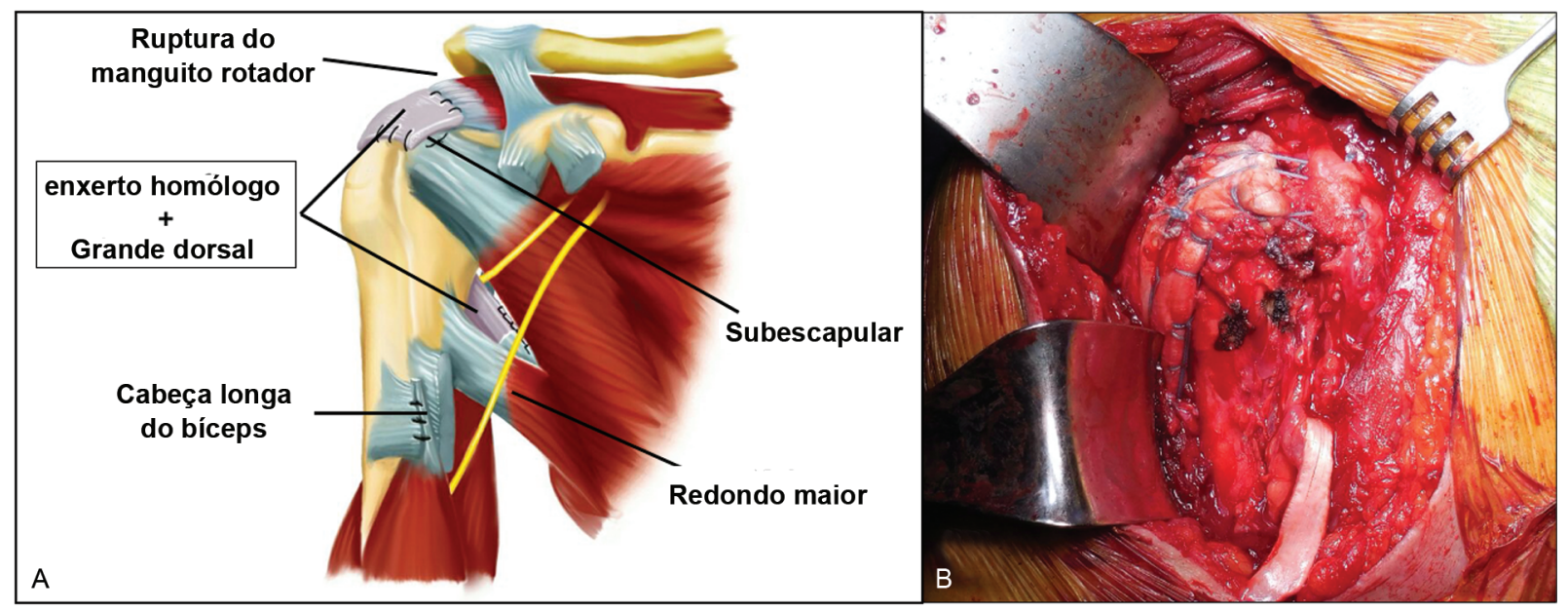

Fig. 3 (A) Ilustração (vista anterior) e (B) fotografia (vista lateral) do aspecto final da cirurgia, onde o tendão do grande dorsal alongado é suturado ao aspecto póstero-lateral do tubérculo maior.

pacientes eram homens e dez $(62,5 \%)$ eram mulheres. A média de idade à cirurgia foi de 65 anos (56-82 anos). Treze cirurgias ocorreram em ombros dominantes (81\%). A etiologia foi traumática em oito pacientes (50\%) e degenerativa em oito $(50 \%)$ (-Tabela 1$)$.

O período médio entre o aparecimento dos sintomas até a cirurgia foi de 32 meses ( 7 a 96 meses), com um período de acompanhamento pós-operatório de no mínimo 12 meses e não superior a 47 (média de 21 meses) (-Tabela 1).

Segundo a classificação de Goutallier do músculo supraespinhoso, três casos eram grau II, oito eram grau III e oito eram grau IV ( - Tabela $\mathbf{1}$ ).

Havia duas lesões do subescapular, ambas limitadas à metade proximal de seus respectivos tendões. Ambas eram eram passíveis de reparo. A metade proximal do ventre muscular desses dois casos foi classificada como grau IV (as metades distais eram grau 0 ) segundo o método de Goutallier ( $\mathbf{- T a b e l a} \mathbf{1}$ ).
Onze ombros foram classificados como grau 1 , três como grau 2, um como grau 3 e um como grau $4 \mathrm{~B}$, segundo o método de Hamada ( - Tabela 1).

Oito dos enxertos usados eram de tendões patelares, seis eram de fáscia lata e um era de tendão do quadríceps. Todos eram enxertos homólogos congelados ( - Tabela $\mathbf{1}$ ).

Os resultados pré-operatórios médios foram comparados aos resultados pós-operatórios médios (-Tabela 2). Além disso, os pacientes foram divididos em subgrupos para comparação dos resultados médios das seguintes variáveis:

- Idade no momento da cirurgia

- Elevação ativa antes da cirurgia (seis indivíduos com pseudoparesia [de $0-90^{\circ}$ ] e dez sem pseudoparesia [> $\left.90^{\circ}\right]$ ) (-Tabela 3).

- Tempo entre o aparecimento dos sintomas e a cirurgia (< 26 meses em dez pacientes e $>35$ meses em seis)

- Tipo de enxerto usado (oito de fáscia lata e sete de tendão patelar). 
Tabela 1 Dados pré-operatórios

\begin{tabular}{|l|l|l|l|l|l|l|l|l|l|l|l|l|}
\hline \multicolumn{2}{|l|}{$\begin{array}{l}\text { Número } \\
\text { do Caso }\end{array}$} & Sexo & Idade & Dominância & Trauma? & $\begin{array}{l}\text { Sintomas } \\
\text { (em meses) }\end{array}$ & SUPRA & INFRA & SUB & HAMADA & Enxerto & $\begin{array}{l}\text { Acompanhamento } \\
\text { (em meses) }\end{array}$ \\
\hline 1 & M & 77 & + & - & 36 & 3 & 3 & 1 & 1 & Patelar & 13 \\
\hline 2 & $\mathrm{M}$ & 56 & - & - & 24 & 2 & 3 & 1 & 2 & Fáscia Lata & 13 \\
\hline 3 & $\mathrm{M}$ & 61 & - & + & 25 & 3 & 4 & 0 & 2 & Patelar & 21 \\
\hline 4 & $\mathrm{~F}$ & 52 & + & + & 10 & 3 & 4 & 1 & 1 & Patelar & 31 \\
\hline 5 & $\mathrm{~F}$ & 63 & + & + & 24 & 4 & 4 & 0 & 1 & Patelar & 43 \\
\hline 6 & $\mathrm{~F}$ & 68 & + & - & 36 & 4 & 4 & 4 & 1 & Fáscia Lata & 12 \\
\hline 7 & $\mathrm{~F}$ & 64 & + & - & 96 & 4 & 4 & 4 & 3 & Patelar & 47 \\
\hline 8 & $\mathrm{~F}$ & 68 & + & + & 52 & 2 & 2 & 2 & $4 \mathrm{~B}$ & Quadríceps & 19 \\
\hline 9 & $\mathrm{M}$ & 68 & + & + & 7 & 3 & 3 & 0 & 1 & Fáscia Lata & 15 \\
\hline 10 & $\mathrm{~F}$ & 59 & + & - & 17 & 2 & 3 & 0 & 1 & Fáscia Lata & 15 \\
\hline 11 & $\mathrm{~F}$ & 67 & + & - & 36 & 3 & 3 & 1 & 2 & Fáscia Lata & 16 \\
\hline 12 & $\mathrm{~F}$ & 56 & + & - & 24 & 4 & 4 & 0 & 1 & Fáscia Lata & 14 \\
\hline 13 & $\mathrm{~F}$ & 61 & + & + & 23 & 3 & 3 & 2 & 1 & Patelar & 28 \\
\hline 14 & $\mathrm{~F}$ & 82 & + & + & 24 & 4 & 4 & 2 & 1 & Patelar & 19 \\
\hline 15 & $\mathrm{M}$ & 67 & - & + & 7 & 3 & 3 & 1 & 1 & Fáscia Lata & 12 \\
\hline 16 & $\mathrm{M}$ & 67 & + & + & 72 & 3 & 3 & 1 & 1 & Fáscia Lata & 15 \\
\hline MÉDIA & & 65 & & & 32 & & & & & & 21 \\
\hline
\end{tabular}

Abreviaturas: Idade, idade no momento da cirurgia; F, feminino; INFRA, infra-espinal; M, masculino; SUB, subescapular; SUPRA, supra-espinal; Trauma?, etiologia traumática?.

- Etiologia da lesão do manguito rotador (atraumática ou traumática).

- Período de acompanhamento pós-operatório ( $<17$ meses em nove pacientes e $>18$ meses em sete indivíduos).

As variáveis contínuas do mesmo grupo foram comparadas por meio de testes $t$ pareados. As variáveis contínuas de grupos diferentes foram comparadas por meio de testes $t$ não pareados. As correlações entre as variáveis contínuas foram determinadas pelo cálculo do coeficiente de Pearson. Os valores de $p \leq 0,05$ foram considerados significativos.

\section{Resultados}

O acompanhamento pós-operatório médio foi de 21 meses (12-47 meses) ( - Tabela 1). A ADM passiva era normal e igual à observada no membro contralateral em todos os ombros, inclusive no caso 8 ( - Tabela $\mathbf{1}$ ), classificado como Hamada 4B.

A elevação ativa média foi de $106^{\circ}$ no pré-operatório $\left(70-104^{\circ}\right)$ e $145^{\circ}$ no pós-operatório $\left(50-160^{\circ}\right)(p<0,001)$. A rotação externa ativa média foi de $30^{\circ}\left(0-60^{\circ}\right)$ no préoperatório e de $54^{\circ}\left(40-70^{\circ}\right)$ no pós-operatório $(p<0,001)$. A rotação interna ativa média foi em L1 no período préoperatório (do glúteo a T5) e em T10 no período pósoperatório $(p=0,017)$ ( - Tabela 2$)$.

De acordo com a pontuação da UCLA, houve $71 \%$ (12 pacientes) de bons resultados ( $\geq 28$ pontos) e $29 \%$ (cinco pacientes) de resultados regulares e ruins ( $\leq 27$ pontos). A pontuação pré-operatória média foi de 11,63 (variação de 8-16), enquanto a pontuação média pós-operatória foi de $27,38$ (variação de 17 a 30$)(p<0,001)$. As comparações dos resultados médios pré e pós-operatórios de cada uma das subpontuações da UCLA foram estatisticamente significativas em todos os casos ( $p<0,001$ para todas as cinco subpontuações) (- Tabela 2).

A comparação dos resultados dos subgrupos revela o seguinte:

- Para idade no momento da cirurgia, tempo entre os primeiros sintomas e a cirurgia, tipo de aloenxerto usado, etiologia da lesão do manguito rotador, e período de acompanhamento pós-operatório: as comparações de ganhos médios (resultados pós-operatórios subtraídos dos resultados pré-operatórios) de todas as variáveis relacionadas à ADM ativa e ao escore da UCLA não mostraram nenhuma diferença estatisticamente significativa.

Nos pacientes com pseudoparesia pré-operatória (elevação média de $75^{\circ}$, variando de $70-90^{\circ}$ ) e nos dez indivíduos sem pseudoparesia (elevação média de $124,5^{\circ}$, variando de $\left.100-140^{\circ}\right)(p<0,001)$ : as comparações dos ganhos médios de ambos os grupos (resultados pós-operatórios subtraídos dos resultados pré-operatórios) não mostraram qualquer diferença estatisticamente significativa em relação à rotação externa, rotação interna ou os seguintes critérios da UCLA: dor, função, força, satisfação, e pontuação total. No entanto, houve diferença estatisticamente significativa em relação à melhora da elevação que foi de $73,33^{\circ}$ em pacientes pseudoparéticos e $18,5^{\circ}$ naqueles não pseudoparéticos 


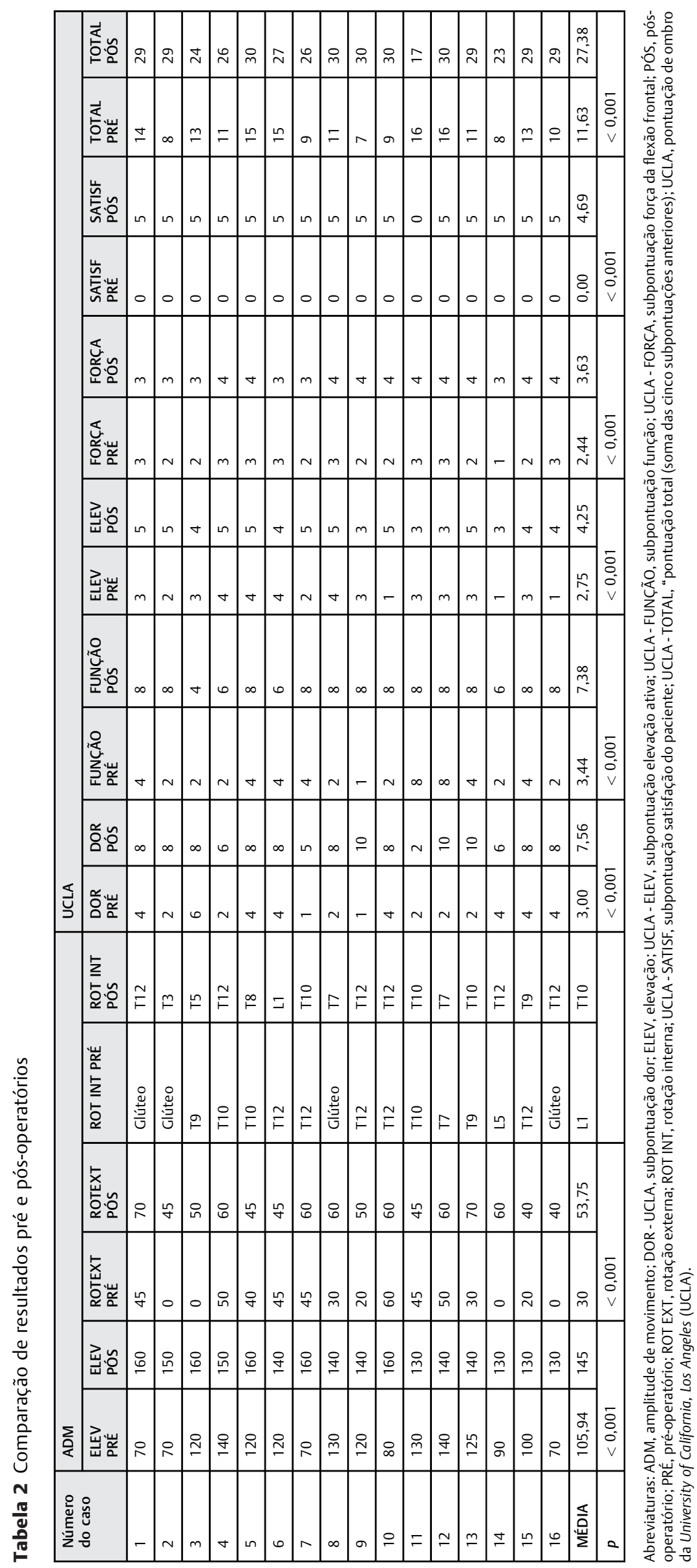


Tabela 3 Comparação de pacientes com pseudoparesia ou não

\begin{tabular}{|c|c|c|c|}
\hline Ganho médio & Pseudoparesia (6 casos) & Sem pseudoparesia (10 casos) & $p$ \\
\hline ADM - FLEX & $73,33^{\circ}$ & $18,5^{\circ}$ & $<0,001^{* * *}$ \\
\hline ADM - ROT EXT & $30,83^{\circ}$ & $19,5^{\circ}$ & 0,27 \\
\hline ADM - ROT INT & $6,17^{\circ}$ & $1,7^{\circ}$ & 0,09 \\
\hline UCLA - DOR & 4 & 4,9 & 0,48 \\
\hline UCLA - FUNÇÃO & 5 & 3,3 & 0,12 \\
\hline UCLA - FLEX & 2,83 & 0,7 & $<0,001^{* * *}$ \\
\hline UCLA - FORÇA & 1,17 & 1,2 & 0,925 \\
\hline UCLA - SATISF & 5 & 4,5 & 0,458 \\
\hline UCLA - TOTAL & 18 & 14,4 & 0,183 \\
\hline ADM - FLEX PRÉ & $75^{\circ}$ & $124,5^{\circ}$ & $<0,001^{* * *}$ \\
\hline ADM - FLEX PÓS & $148,33^{\circ}$ & $143^{\circ}$ & 0,41 \\
\hline
\end{tabular}

Abreviaturas: ADM, amplitude de movimento; UCLA - DOR, subpontuação dor; FLEX, flexão frontal; UCLA - FLEX, subpontuação flexão frontal ativa; UCLA - FUNÇÃO, subpontuação função; UCLA - FORÇA, subpontuação força da flexão frontal; PÓS, pós-operatório; PRÉ, pré-operatório; ROT EXT, rotação externa; ROT INT, rotação interna; UCLA - SATISF, subpontuação satisfação do paciente; UCLA - TOTAL, pontuação total (soma das cinco subpontuações anteriores); UCLA, pontuação de ombro da University of California, Los Angeles (UCLA).

${ }^{* * *}$ Diferença estatisticamente significativa.

$(p<0,001)$, bem como na subpontuação da elevação da UCLA, que foi de 2,83 pontos em pacientes pseudoparéticos e 0,7 naqueles não pseudoparéticos $(p<0,001)$. Como mostra a última linha da - Tabela 3, essa diferença no ganho de elevação foi capaz de promover resultados estatisticamente equivalentes em ambos os grupos, que foi de $148,33^{\circ} \mathrm{em}$ pacientes pseudoparéticos e $143^{\circ}$ em pacientes não pseudoparéticos $(p=0,41)$, mostrando, portanto, que a pseudoparesia não é preditora de pior resultado ( - Tabela 3 ).

\section{Discussão}

Os principais objetivos da transferência do TGD para o tratamento de lesões irreparáveis do manguito rotador são melhora da ADM ativa, dor, função e força do ombro, bem como da satisfação do paciente. Nossos resultados demonstram que todos esses objetivos foram alcançados.

As melhoras obtidas de rotação externa, rotação interna e elevação ativas foram semelhantes às observadas em outros trabalhos publicados, ${ }^{1,2,9-11,19,20}$ com ganhos médios de $39^{\circ}$ $(p<0,001), 3$ níveis vertebrais $(p=0,017)$ e $24^{\circ}(p<0,001)$, respectivamente ( $\mathbf{-}$ Tabela $\mathbf{2}$ ).

Essa técnica parece ser eficaz na melhora do alívio da dor, considerando o ganho médio de 4,53 pontos $(p<0,001)$ no critério dor da UCLA (que varia de 0 -10), além de melhorar a função do ombro, como mostra o ganho médio de 3,71 pontos $(p<0,001)$ no critério função da UCLA (que varia de $0-10$ ). Esses dois resultados foram semelhantes aos observados na maioria dos outros trabalhos, ${ }^{1,2,9-11,19,20}$ como o de Grimberg et al., ${ }^{8}$ que relatou um ganho médio de 28,4 pontos (de 37,0-65,4) na pontuação total de Constant e de 10,9 pontos (de 1,7 antes da cirurgia para 12,6 após o procedimento) na subpontuação dor.

Todos os pacientes apresentaram melhoras na força de elevação exceto nos dois primeiros casos, nos quais a força permaneceu a mesma. No entanto, a força não voltou ao normal (grau 5) em qualquer caso, continuando em grau 3 em seis pacientes e grau 4 em cinco pacientes. Havia a preocupação de que o alongamento do tendão transferido pudesse prejudicar a força de elevação do ombro. No entanto, a análise da literatura mostra que a recuperação da força foi muito semelhante à observada em outras séries (sem utilização de enxerto), ${ }^{2,8-11,19,30}$ como relatado por Gerber et al., ${ }^{30}$ que obtiveram melhora da força média de $0,9 \mathrm{~kg}$ no pré-operatório para $1,8 \mathrm{~kg}$ no pós-operatório $(p<0,0001)$ nos 65 ombros assim avaliados

Surpreendente e diferentemente dos achados de Iannotti et al. ${ }^{10}$ (técnica convencional), a pseudoparesia foi resolvida em todos os casos. Acreditamos que isso tenha ocorrido porque os enxertos tendíneos utilizados eram mais espessos do que o tendão do grande dorsal nativo e, portanto, também podem ter funcionado como espaçador subacromial. Assim, seria mais fácil manter a estabilidade do centro de rotação do ombro, favorecendo a função de elevação do deltoide.

De acordo com a pontuação da UCLA, obtivemos $71 \%$ de bons resultados e $29 \%$ de regulares e ruins. Tais achados são semelhantes aos da literatura, que mostra cerca de 30\% de resultados insatisfatórios, como relatam Kany et al. ${ }^{21}$ Apesar desses resultados insatisfatórios, acreditamos que esses pacientes terão menos complicações em médio e longo prazo, visto que essa técnica pode estar menos sujeita a novas rupturas, visto que Kany et al. ${ }^{12}$ mostraram de 15 a $46 \%$ de rupturas de transferências artroscópicas do TGD, ocorridas com somente três meses de acompanhamento pósoperatório.

A artroplastia reversa do ombro (ARO) é, sem dúvidas, o último recurso cirúrgico após a falha de uma transferência de tendão. Porém, a ARO requer que o deltoide seja funcional, que é a última unidade motora disponível capaz de gerar vetores de força para cima que resultariam em elevação. ${ }^{2}$ Assim, é altamente recomendado evitar qualquer violação cirúrgica do deltoide, como em abordagens superiores (em 
"golpe de sabre"). Portanto, há apenas três abordagens cirúrgicas que poupam o deltoide: a abordagem posterior única, a abordagem axilar seguida de abordagem artroscópica e a abordagem anterior única.

A maior desvantagem da abordagem posterior única é impedir o tratamento de lesões na cabeça longa do bíceps e no tendão subescapular.

Da mesma forma, a abordagem axilar não permite 0 reparo primário da ruptura do manguito rotador, que sempre deve ser tentado antes de qualquer transferência de tendão.

As vantagens da abordagem deltopeitoral única são o fato de ser habitual e permitir o reparo primário da ruptura do manguito rotador, se possível, e o fácil tratamento das lesões da cabeça longa do bíceps e do subescapular. Sua única desvantagem é a impossibilidade de dissecção do ventre do músculo grande dorsal e, portanto, sua tração para a parte superior da cabeça do úmero. Como esse tendão é extremamente delgado e friável, porém, recomenda-se o reforço de sua inserção no tubérculo maior, já que sua ruptura pós-operatória, geralmente nos dois primeiros anos após a cirurgia, é um dos poucos fatores preditivos estatisticamente comprovados de maus resultados funcionais. ${ }^{2}$ Portanto, acreditamos que o uso de enxerto tendíneo pode resolver os dois problemas ao mesmo tempo: permite o uso da abordagem única (que protege o deltoide) e reforça a inserção do tendão no tubérculo maior.

Existem três possíveis complicações: (1) a fragilidade entre o enxerto e o tendão nativo; (2) a capacidade de cicatrização do enxerto homólogo ao osso; e (3) a possibilidade de alongamento excessivo do TGD, reduzindo muito a força final resultante no úmero. Embora nosso trabalho não tenha avaliado diretamente essas três possíveis complicações, elas não parecem ocorrer, uma vez que todos os pacientes apresentaram melhora na subpontuação força da UCLA, já discutida.

Como objetivo secundário, avaliamos se alguma variável pré-operatória (idade, pseudoparesia, duração dos sintomas e etiologia da ruptura do manguito rotador), intraoperatória (o tipo de enxerto homólogo usado) e pós-operatória (período de acompanhamento) influenciaram os resultados. Apesar do número limitado de pacientes, os resultados mostraram que nenhuma dessas variáveis influenciou os resultados de forma significativa.

Vale destacar os casos 6 e 7, que apresentavam lesão completa da metade proximal do subescapular. Nos dois casos, as lesões foram reparadas (simultaneamente à transferência) e ambos apresentaram melhora notável no movimento do ombro e na pontuação da UCLA ( - Tabelas 1 e 2). Da mesma forma, os casos 7 e 8 tinham degeneração articular avançada (Hamada 3 e 4B, respectivamente) e apresentaram recuperação da $A D M$ do ombro e melhora notável no escore da UCLA ( - Tabelas 1 e 2 ).

Por fim, vale destacar as deficiências deste trabalho: sendo uma análise retrospectiva de casos, não houve grupo controle e, portanto, todas as comparações foram feitas com achados pré-operatórios ou de outros trabalhos publicados, com utilização de metodologias diferentes. Além disso, embora a perda amostral tenha sido de apenas 20\% (ou quatro casos), o número total de casos avaliados foi pequeno, comprometendo o poder da análise estatística.

\section{Conclusão}

No acompanhamento em curto e médio prazo, a técnica estudada foi eficaz no tratamento de lesões póstero-superiores irreparáveis do manguito rotador, com 71\% de resultados satisfatórios. Nenhuma das variáveis avaliadas, inclusive a pseudoparesia pré-operatória, influenciou os resultados obtidos.

Suporte Financeiro

Não houve suporte financeiro de fontes públicas, comerciais, ou sem fins lucrativos.

Conflito de Interesses

Os autores declaram que não há conflito de interesses.

\section{Referências}

1 Gerber C. Latissimus dorsi transfer for the treatment of irreparable tears of the rotator cuff. Clin Orthop Relat Res 1992;(275): $152-160$

2 El-Azab HM, Rott O, Irlenbusch U. Long-term follow-up after latissimus dorsi transfer for irreparable posterosuperior rotator cuff tears. J Bone Joint Surg Am 2015;97(06):462-469

3 Mulieri P, Dunning P, Klein S, Pupello D, Frankle M. Reverse shoulder arthroplasty for the treatment of irreparable rotator cuff tear without glenohumeral arthritis. J Bone Joint Surg Am 2010;92(15):2544-2556

4 Gerber C, Canonica S, Catanzaro S, Ernstbrunner L. Longitudinal observational study of reverse total shoulder arthroplasty for irreparable rotator cuff dysfunction: results after 15 years. J Shoulder Elbow Surg 2018;27(05):831-838

5 Westermann RW, Pugely AJ, Martin CT, Gao Y, Wolf BR, Hettrich CM. Reverse Shoulder Arthroplasty in the United States: A Comparison of National Volume, Patient Demographics, Complications, and Surgical Indications. Iowa Orthop J 2015;35:1-7

6 Checchia C, Domos P, Grimberg J, Kany J. Current Options in Tendon Transfers for Irreparable Rotator Cuff Tears. JBJS Rev 2019; 7(02):e6

7 Castricini R, Longo UG, De Benedetto M, et al. ArthroscopicAssisted Latissimus Dorsi Transfer for the Management of Irreparable Rotator Cuff Tears: Short-Term Results. J Bone Joint Surg Am 2014;96(14):e119

8 Grimberg J, Kany J, Valenti P, Amaravathi R, Ramalingam AT. Arthroscopic-assisted latissimus dorsi tendon transfer for irreparable posterosuperior cuff tears. Arthroscopy 2015;31(04):599-607.e1

9 Habermeyer P, Magosch P, Rudolph T, Lichtenberg S, Liem D. Transfer of the tendon of latissimus dorsi for the treatment of massive tears of the rotator cuff: a new single-incision technique. J Bone Joint Surg Br 2006;88(02):208-212

10 Iannotti JP, Hennigan S, Herzog R, et al. Latissimus dorsi tendon transfer for irreparable posterosuperior rotator cuff tears. Factors affecting outcome. J Bone Joint Surg Am 2006;88(02):342-348

11 Irlenbusch U, Bracht M, Gansen H-K, Lorenz U, Thiel J. Latissimus dorsi transfer for irreparable rotator cuff tears: a longitudinal study. J Shoulder Elbow Surg 2008;17(04):527-534

12 Kany J, Sekaran P, Grimberg J, et al. Risk of latissimus dorsi tendon rupture after arthroscopic transfer for posterior superior rotator cuff tear: a comparative analysis of 3 humeral head fixation techniques. J Shoulder Elbow Surg 2020;29(02):282-290

13 Moursy M, Forstner R, Koller H, Resch H, Tauber M. Latissimus dorsi tendon transfer for irreparable rotator cuff tears: a modified 
technique to improve tendon transfer integrity. J Bone Joint Surg Am 2009;91(08):1924-1931

14 Mihata T, Lee TQ, Fukunishi K, et al. Return to Sports and Physical Work After Arthroscopic Superior Capsule Reconstruction Among Patients With Irreparable Rotator Cuff Tears. Am J Sports Med 2018;46(05):1077-1083

15 Burkhart SS, Hartzler RU. Superior Capsular Reconstruction Reverses Profound Pseudoparalysis in Patients With Irreparable Rotator Cuff Tears and Minimal or No Glenohumeral Arthritis. Arthroscopy 2019;35(01):22-28

16 Aibinder WR, Elhassan BT. Lower trapezius transfer with Achilles tendon augmentation: indication and clinical results. Obere Extrem 2018;13(04):269-272

17 Elhassan BT, Wagner ER, Werthel JD. Outcome of lower trapezius transfer to reconstruct massive irreparable posterior-superior rotator cuff tear. J Shoulder Elbow Surg 2016;25(08):1346-1353

18 Gerber C, Rahm SA, Catanzaro S, Farshad M, Moor BK. Latissimus dorsi tendon transfer for treatment of irreparable posterosuperior rotator cuff tears: long-term results at a minimum follow-up of ten years. J Bone Joint Surg Am 2013;95(21):1920-1926

19 Grimberg J, Kany J. Latissimus dorsi tendon transfer for irreparable postero-superior cuff tears: current concepts, indications, and recent advances. Curr Rev Musculoskelet Med 2014;7(01):22-32

20 Warner JJ. Management of massive irreparable rotator cuff tears: the role of tendon transfer. Instr Course Lect 2001;50:63-71

21 Kany J, Grimberg J, Amaravathi RS, Sekaran P, Scorpie D, Werthel JD. Arthroscopically-Assisted Latissimus Dorsi Transfer for Irreparable Rotator Cuff Insufficiency: Modes of Failure and Clinical Correlation. Arthroscopy 2018;34(04):1139-1150

22 Costouros JG, Espinosa N, Schmid MR, Gerber C. Teres minor integrity predicts outcome of latissimus dorsi tendon transfer for irreparable rotator cuff tears. J Shoulder Elbow Surg 2007;16(06): 727-734

23 Lehmann LJ, Cafaltzis K, Hünnebeck S, Moursy M. Are there any prognostic prediction parameters (PPPs) in the treatment of the massive rotator cuff tear with latissimus dorsi transfer? Latissimus dorsi transfer in massive rotator cuff tears. Acta Chir Orthop Traumatol Cech 2013;80(02):125-130

24 Sher JS, Iannotti JP, Warner JJ, Groff Y, Williams GR. Surgical treatment of postoperative deltoid origin disruption. Clin Orthop Relat Res 1997;(343):93-98

25 Miyazaki AN, Checchia CS, de Castro Lopes W, Fonseca Filho JM, Sella GDV, da Silva LA. Latissimus Dorsi Tendon Transfer using Tendinous Allograft for Irreparable Rotator Cuff Lesions: Surgical Technique. Rev Bras Ortop (Sao Paulo) 2019;54(01): 99-103

26 Fuchs B, Weishaupt D, Zanetti M, Hodler J, Gerber C. Fatty degeneration of the muscles of the rotator cuff: assessment by computed tomography versus magnetic resonance imaging. J Shoulder Elbow Surg 1999;8(06):599-605

27 Hamada K, Fukuda H, Mikasa M, Kobayashi Y. Roentgenographic findings in massive rotator cuff tears. A long-term observation. Clin Orthop Relat Res 1990;(254):92-96

28 Codsi M. Clinical evaluation of shoulder problems. In: Rockwood CA Jr, Matsen FA 3rd, Wirth MA, Lippitt SB, editors. The shoulder. 4th ed. Philadelphia, PA: Saunders Elsevier; 2009:148-176

29 Ellman H, Hanker G, Bayer M. Repair of the rotator cuff. End-result study of factors influencing reconstruction. J Bone Joint Surg Am 1986;68(08):1136-1144

30 Gerber C, Maquieira G, Espinosa N. Latissimus dorsi transfer for the treatment of irreparable rotator cuff tears. J Bone Joint Surg Am 2006;88(01):113-120 